\title{
Percorsi pratici per intraprendere un programma di dialisi monosettimanale combinato con una nutrizione ipoproteica personalizzata
}

Giornale di Tecniche Nefrologiche e Dialitiche 2018, Vol. 30(2) 74-79

(C) The Author(s) 2018

Article reuse guidelines:

sagepub.com/journals-permissions

DOI: $10.1177 / 0394936218760823$

journals.sagepub.com/home/gtn

\author{
Piergiorgio Bolasco', Stefano Murtas², Gabriella Concas², Andrea Galfré2
}

\begin{abstract}
Practical paths to undertake a one-week dialysis program combined with a nutrition personalized hypoproteics

The aim of this article is to provide detailed information to nephrologists on how to apply the combined diet dialysis program (CDDP) in selected patients. Patients are evaluated by urea nitrogen appearance, which allows the sustainability and compliance of patients on $0.6 \mathrm{~g} / \mathrm{kg}$ per day low-protein diet to be verified by calculating the protein catabolic rate at a metabolic steady state. The combined action of nutrition and less frequent hemodialysis may preserve residual renal function with a further chance of achieving greater excretion of the protein-bound uremic toxins, thereby obtaining a positive phosphate balance. This paper describes all fundamental steps and calculations, although a greater clinical commitment is required to achieve a tailored therapeutic protocol such as Combined Diet Dialysis Program (CDDP).
\end{abstract}

\section{Keywords}

Combined Diet Dialysis Program, once-weekly dialysis, diet program

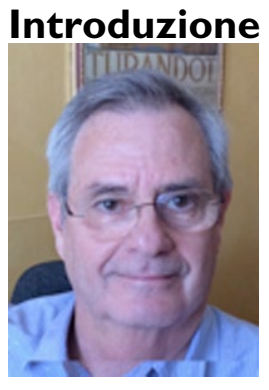

Come è noto la terapia nutrizionale può essere un valido provvedimento per consentire di procrastinare il momento di inizio del trattamento sostitutivo $^{1,2}$ oppure ridurre la necessità e la frequenza di dose dialitica $^{3}$ e quindi per permettere al paziente un approccio più morbido ed accettabile nel momento di transizione dalla terapia conservativa alla dialisi.

$\mathrm{Nel} 1994$ il gruppo di Locatelli, ${ }^{4}$ riprendendo lo schema di Giovannetti et al..$^{5}$ e le esperienze di Mitch ${ }^{6}$ proposero l'integrazione di una dialisi monosettimanale ad una dieta ipoproteica di 0,3-0,4 g/kg/die supplementata con aminoacidi essenziali e chetoanaloghi. Questo programma fu denominato Integrated dialysis diet program (IDDP). ${ }^{7}$

La difficile applicabilità di una dieta cosi severa, l'elevato numero di drop-out e la preoccupazione di indurre malnutrizione portarono alla conclusione che un programma che combini una forte limitazione nutrizionale ipoproteica insieme ad una dialisi monosettimanale non è possibile applicarlo in larga scala, ma può, implementando alcuni parametri della IDDP, considerarsi una valida opzione da proporre a pazienti attentamente selezionati e particolarmente motivati. ${ }^{8}$ Facendo quindi tesoro delle esperienze precedenti e cercando di correggere alcuni punti di debolezza, abbiamo riproposto un programma denominato Combined diet dialysis program (CDDP). ${ }^{9}$

Questo articolo ha lo scopo di proporre ai Nefrologi una antica ma nuova metodologia ormai ben consolidata nei nostri pazienti da oltre 25 anni. L'obiettivo di questo lavoro è di definire quali minimi requisiti sarebbero necessari in un centro di nefrologia e dialisi per sottoporre ai pazienti in fase pre-dialitica una metodica alternativa, seppur non definitiva, che può sfociare, nell'outcome dei pazienti in CDDP, in un trapianto renale senza aver subito eccessivi

'Già Direttore della S.C. Territoriale di Nefrologia e Dialisi ASL di Cagliari, Membro del Gruppo di Studio sul trattamento conservativo della insufficienza renale cronica della Società Italiana di Nefrologia ${ }^{2}$ S.C. Territoriale di Nefrologia e Dialisi ASL di Cagliari, Cagliari

\section{Corrispondenza:}

Piergiorgio Bolasco, MD, Via Albert Einstein 21, 09I26 Cagliari, Italia

E-mail: pg.bolasco@gmail.com 
contatti con il trattamento emodialitico che per le sue caratteristiche microinfiammatorie, pro-ossidative ${ }^{10,11}$ deve considerarsi dannoso al mantenimento della funzione renale residua (FRR) non può definirsi biocompatibile con l'organismo umano nonostante l'utilizzo di membrane e materiali sempre meno bio-incompatibili. ${ }^{12,13}$ Nei pazienti anziani le permanenze in CDDP possono superare agevolmente i 2-3 anni con una buona stabilità dello stato metabolico insieme ad una gratificazione del paziente che acquisisce una migliore qualità di vita e acquista ulteriore indipendenza dal trattamento stesso.

\section{Cenni metodologici introduttivi}

Rispetto agli studi degli anni ottanta e novanta ${ }^{4,5}$, la CDDP prevede una dieta ipoproteica meno severa $(0,6 \mathrm{~g} / \mathrm{kg} / \mathrm{die})$, sempre ipofosforica e normocalorica, con dieta libera in proteine il giorno della dialisi per coprire l'aumentato fabbisogno azotato legato alle perdite di proteine e aminoacidi dovute alla seduta di emodialisi. ${ }^{14}$

I pazienti reclutabili devono essere in stato metabolico stazionario e conservare una funzione renale residua tra 5 e $12 \mathrm{~mL} / \mathrm{min} / 1,73 \mathrm{mq}$. Condizione indispensabile è la motivazione e la buona aderenza alla terapia nutrizionale prescritta. Inoltre anche l'uso di membrane meno bioincompatibili ${ }^{15}$ e la buona qualità dell'acqua di dialisi ${ }^{16}$ assicurano oggi minore microinfiammazione e proossidazione intra-sessione.

\section{Materiale e metodi}

\section{Percorso pre-dialisi}

Paziente naïve (proveniente da altro centro o ex-novo). Il paziente viene preso in carico dall'ambulatorio pre-dialisi o nell'ambulatorio di un nefrologo esperto nella gestione dell'uremia terminale per effettuare un corretto inquadramento dello stadio di insufficienza renale cronica (CKD), della FRR ed indurre una stabilizzazione metabolica. Dopo aver proposto al paziente le varie scelte di terapia sostitutiva e quest'ultimo opta per la "dialisi monosettimanale" si prescrive la terapia nutrizionale sotto descritta. Il paziente o colui che effettua la scelta degli alimenti per i pasti dovrebbe essere informato sulla composizione degli alimenti confezionati a particolare contenuto proteico-fosforico (specie quelli contenenti additivi).

Opzionale: ambulatorio con nefro-nutrizionista, dietista assegnata al reparto, counseling medico-infermieristico del paziente e della famiglia. Tutto ciò può essere anche effettuato da Operatori con alte capacità comunicative di rassicurazione e riferimento.

Paziente già seguito da tempo nei propri ambulatori. Dovrebbe essere già stabilizzato e dovrebbe o dovrà fare i percorsi già sopradescritti.
Allestimento accesso vascolare. Si può iniziare anche con un catetere venoso centrale (CVC) efficiente in attesa della maturazione di una fistola artero-venosa (FAV).

\section{Criteri di idoneità}

Caratteristiche che permettono di iniziare un programma di CDDP:

- FRR: VFG $5-12 \mathrm{~mL} / \mathrm{min} / 1,73 \mathrm{mq}$ (volume di diuresi/die medio settimanale $>1.0-1.5 \mathrm{~L} / \mathrm{die})$;

- non segni di ritenzione idrosalina e/o accertata responsività alla terapia diuretica;

- incremento inter dialitico ponderale tra una dialisi e la successiva 3 - $5 \%$ del peso secco;

- costante aderenza alla terapia nutrizionale prescritta;

- potassiemia stabilmente controllabile $<5.5-6.0$ $\mathrm{mEq} / \mathrm{L}$;

- fosfatemia stabilmente controllabile $<5.5-6.0 \mathrm{md} /$ $\mathrm{dL}$;

- assenza di condizioni ipercataboliche e buono stato di nutrizione;

- percezione da parte del paziente di una buona qualità di vita;

- emoglobina nei range secondo linea guida o facilmente correggibile con Agenti Stimolanti l'Eritropoiesi (ASEs).

- assenza di patologie co-morbide che richiedano frequenti ospedalizzazioni;

- accesso vascolare che consenta flussi di $300 \mathrm{~mL} /$ min o più con assenza di ricircolo

- Single pool Kt/V $>1,4$ o equilibrated Kt/V $>1,2$;

- durata trattamento extracorporeo $>4$ ore;

- assenza o molto rari episodi di ipotensioni sintomatiche durante la sessione emodialitica;

- eccellente qualità dell'acqua per dialisi sotto il profilo batteriologico e/o endotossinico;

- preferenza di metodiche dialitiche con dialisato senza acetato;

- utilizzo di membrane sintetiche;

- BMI compreso tra 25 e 28;

\section{Esclusioni}

- qualsiasi ragione clinica che possa far sospettare un'uremia non ben controllata;

- cattiva compliance alimentare del paziente, la quale può già estrapolarsi dal calcolo dall'urea nitrogen appearance (UNA) ma già è sospetta se la BUN predialitica è persistentemente $>100-120 \mathrm{mg} / \mathrm{dL}$;

- eccessivi incrementi ponderali interdialitici (> 5-6\% proporzionalmente al peso secco);

- scompenso cardiaco latente o conclamato non presente all'inizio del programma; 
- iperparatiroidismo terziario incontrollabile, presenza di disturbi neurologici centrali o periferici.

\section{Prescrizione nutrizionale}

Quantità di proteine pari a $0,6 \mathrm{~g} / \mathrm{kg}(30-40 \%$ ad alto valore biologico), contenuto fosfati $<800 \mathrm{mg}$, contenuto di potassio 30-80 mEq/die; contenuto di sodio: $40-80 \mathrm{mEq} /$ die; contenuto calorico > $30 \mathrm{Kcal} /$ die. Apporto di grassi: < $40 \%$ dell'apporto calorico totale. In caso di presenza di alterazioni del metabolismo lipidico si devono individuare eventuali errori alimentari e/o correggere le alterazioni lipidiche.

Il pasto del giorno di dialisi è libero tenendo conto di evitare un'assunzione proteica $>1,4-1,5 \mathrm{~g} / \mathrm{kg} / \mathrm{die}$ esplicitando le dovute istruzioni al paziente sul contenuto di potassio di alcuni alimenti.

E' importante integrare l'alimentazione con miscele di aminoacidi prive di fosfati e composti azotati con prevalenza di aminoacidi essenziali e ramificati a causa della difficoltà di sintesi proteica causata dalla disbiosi del microbiota presente nello status uremico avanzato.

Si consideri che il paziente in CDDP conserva una importante fosfaturia, kaliuresi, sodiuria e talora proteinurie superiori ai $2 \mathrm{~g} / \mathrm{die}$. Occorre tenere conto, in casi sporadici, di un aggiustamento nella prescrizione proteica in caso di severa proteinuria.

\section{Metodologia dialitica}

La scelta ricade sulla metodica che possa indurre il minor numero di ipotensioni e che assicuri l'adequatezza già descritta; occorre evitare eccessive disidratazioni. Il peso forma è puramente indicativo e può essere stabilito dall'osservazione clinica (visite obbligatorie prima dell'inizio dialisi) e se possibile da una periodica valutazione bioimpedenziometrica. Il paziente può agevolmente terminare la dialisi con un peso forma $>300$ $500 \mathrm{~g}$ poiché egli spesso urina subito dopo il trattamento o perde l'eccesso di liquidi nelle 24 ore successive al trattamento. Se il paziente arriva in dialisi sotto il peso forma, va reidratato durante la seduta per ripristinare il peso forma ideale, eccetto che nei casi di dimagrimento dovuti ad una inadeguatezza del paziente ad alimentarsi con le quantità proteico-caloriche prescritte.

\section{Terapie farmacologiche}

Non è necessario che la dose di furosemide per il mantenimento della diuresi superi $1,5 \mathrm{~g} /$ die. Oltre tali dosaggi non ci si deve aspettare una maggiore risposta da parte dei nefroni residui. Gli ACE- inibitori non sono controindicati. È comunque necessario un adeguato controllo dei livelli di pressione arteriosa nei range di normalità. Occorre necessariamente evitare l'utilizzo di anti-infiammatori/antidolorifici del gruppo FANS. ̇̀ necessario un supplemento vitaminico dopo la dialisi ed a domicilio con acido folico e vitamine del gruppo B.

\section{Sorveglianza emato-chimica}

Seguire i ritmi di controllo usuali utilizzati dal centro dialisi. In aggiunta:

- Mensilmente: protidemia totale ed albuminemia.

- Ogni sei mesi: assetto lipidico, C3, transferrina.

- Nei ritmi sopradescritti il giorno della dialisi: clearance dell'urea e della creatinina, $\mathrm{Na}, \mathrm{K}, \mathrm{Ca}, \mathrm{P}$, emogasanalisi, $\mathrm{Hb}$ ematici. Opzionali: sodiuria, calciuria, fosfaturia, potassiuria e proteinuria delle 24 ore.

Occorre utilizzare una apposita scheda da inserire nella cartella di questi pazienti onde registrare tutti i parametri ematochimici, volume diuresi, clearance ed altri rilievi urinari, parametri per il calcolo dell'UNA e quindi il calcolo del protein catabolic rate (PCR ricavata) che indirettamente ma con buona precisione ci rende edotti dell'assunzione proteica del paziente. Questa PCR ricavata (PCRr) sarà da confrontare con la cosiddetta $\mathrm{PCR}$ attesa $(\mathrm{PCRa})$ pari alla prescrizione proteica in $\mathrm{g} / \mathrm{Kg} / \mathrm{die}$.

\section{Calcolo della funzione renale residua (FRR)}

Il gruppo di Locatelli nelle fasi iniziali dell'IDDP effettuava tutti o quasi tutti i giorni extra-dialisi la raccolta delle urine delle 24 ore. La nostra procedura di CDDP utilizza la media delle clearance $(\mathrm{Kr})$ dell'urea e creatinina su tre raccolte settimanali $(\mathrm{Kr}=$ media $\mathrm{Kr}$ urea/creatinina $)$. Si consiglia di evitare l'utilizzo dell'MDRD e/o del CKD/ EPI per valutare la FRR. La sequenza delle $\mathrm{Kr}$ prevede come ultima la raccolta delle urine 24 ore il giorno precedente l'emodialisi.

RITMO RACCOLTE: dipende dal giorno in cui il paziente è sottoposto a trattamento emodialitico (sia di mattina che di pomeriggio) anche in funzione degli accordi con i laboratori di riferimento. Occorrerà effettuare tre raccolte delle 24 ore. Infatti i pazienti andrebbero allocati nei giorni mid-week onde "incastrarli" con pazienti in ritmo bisettimanale. Sono da evitare raccolte urine nelle 12 ore successive alla dialisi sia per la possibile oliguria, sia per l'ipercatabolismo indotto dalla dialisi e sia per la dieta libera il giorno stesso del trattamento. Ovviamente il paziente dovrà recarsi al centro di cura per portare la raccolta delle urine ed eseguire il prelievo a digiuno.

L'occasione del giorno dialisi può essere propizia per controllare peso e pressione del paziente e/o, una volta ottenuti i risultati degli esami onde eventualmente richiamarlo al centro dialisi per i necessari provvedimenti (dialitici o farmacologici). 
Nei primi tre mesi di CDDP tali controlli si effettuano il più possibile equidistanti una volta al mese. Successivamente, se il paziente viene giudicato stabile e collaborante, ogni 2-3 mesi a seconda del trend di declino della funzione renale residua.

È possibile assistere ad un recupero della FRR e non è raro effettuare dialisi mono-quindicinali oppure sospendere la CDDP e reinserire il paziente nei controlli ambulatoriali.

Il paziente in CDDP conserva generalmente una buona diuresi e non riserva sorprese (come pericolose e/o subdole iperidratazioni, iperpotassiemia).

\section{Valutazione della collaborazione dietetica}

Occorre sempre considerare che il programma CDDP è prioritariamente un programma dietetico e la dialisi è fondamentale come momento di controllo e di riequilibrio compartimentale onde anche giustificare la scelta "non del tutto conservativa" con livelli di insufficienza renale cronica avanzata. La dialisi monosettimanale è un riferimento fondamentale di garanzia e validità del programma. Essa si svolge utilizzando la formula di Maroni e Mitch. ${ }^{17}$

1) Valutazione dell'acqua corporea ( $\mathrm{Vb}$ ) ove l'urea si distribuisce. Tutte le formule sono valide; abitualmente viene utilizzata la formula di Watson ${ }^{18}$ ma possono essere utilizzate numerose alternative ritrovabili nel WEB e magari più personalizzabili per l'età, sesso e tipologia del paziente.

2) Protein catabolic rate ( $\mathrm{PCR}$ ricavata, $\mathrm{PRCr}$ )

$$
P C R r=6,25 \quad \frac{d N+U U N+N U N}{T i}
$$

Ove:

$d N=$ accumulo di azoto ureico nel volume di acqua corporea $(\mathrm{Vb})$

$d N$ si ricava $=d B U N \times V b \times B U N p \times d B W$

$d B W$ è la eventuale variazione di peso in positivo tra il peso pre-dialitico nelle due dialisi successive

$d B U N$ è rappresentata dalla variazione della BUN nel nell'intervallo $T i$

$B U N p$ è rappresentato dai livelli di BUN a fine dell'intervallo Ti (livelli di BUN prelevati quando il paziente porta la raccolta delle urine 24 ore).

$T i=$ intervallo dialitico

$U U N=$ azoto ureico urinario escreto nelle 24 ore di raccolta da convertire in $\mathrm{g}$

$N U N$ : Azoto non ureico eliminato nell'intervallo $T i$ (è una quota fissa indipendentemente dell'entità dell'uremia ed equivale a $31,3 \mathrm{mg} / \mathrm{Kg} /$ die. $^{17}$

\section{Esempio pratico su un'unica raccolta di 24 ore}

Uomo: $70 \mathrm{~kg}$ con $\mathrm{Vb}=42 \mathrm{~L}$; peso dialisi $1=70,000 \mathrm{~kg}$; peso dialisi $2=70,600 \mathrm{~kg}$

UUN: $205 \mathrm{mg} / \mathrm{dL}$; diuresi $24 \mathrm{~h}$ : 1,5 L; UUN $24 \mathrm{~h}=3,07 \mathrm{~g}$.

NUN: $31,3 \mathrm{mg} \times 70=2,19 \mathrm{~g} / \mathrm{die}$

BUNp $1^{\circ}: 76 \mathrm{mg} / \mathrm{dL}=760 \mathrm{mg} / \mathrm{L}=0,76 \mathrm{~g} / \mathrm{L}$

BUNp $2^{\circ}$ finale: $83 \mathrm{mg} / \mathrm{dL}=830 \mathrm{mg} / \mathrm{L}=0,83 \mathrm{~g} / \mathrm{L}$

Intervallo interdialitico (Ti): 1 giorno

$d B U N=0,83-0,76=0,07 \mathrm{~g}$ (di accumuli nel $\mathrm{Vb}$ )

$d B W=0,6 L$

$d N=0,07 \times 42 \times 0,83 \times 0,6=1,464 \mathrm{~g} / 24 \mathrm{~h}$

$P C R=6.25 X \underline{1,464+3,07+2,19}$ uguale $6,72 \mathrm{~g} \mathrm{di}$

azoto $X$ 6,25 (fattore di conversione di $N$ in proteine)

1 (giorno)

$P C R r=$ assunzione proteica $(P C R$ ricavata $)=46,82 \mathrm{~g} /$ die

dieta prescritta $(\mathrm{PCR}$ ) in $\mathrm{g} / \mathrm{Kg} /$ die prescritta $=0,6 \mathrm{X}$ $70=42 \mathrm{~g}=42 \mathrm{~g} /$ die - PCRa di 42,02; Variazione $+0,02 \mathrm{~g}$ di proteine/die

(valutazione ottima compliance dietetica)

È opportuno che il paziente effettui il prelievo del BUNp il giorno successivo alla dialisi a prescindere da una possibile coda dell'ipercatabolismo proteico della dialisi del giorno precedente e da quella del pasto libero consentito al paziente; generalmente non si ha una eccessiva contrazione della diuresi delle 24 ore. Ciononostante riteniamo attendibili le tre raccolte settimanali ed il calcolo non è particolarmente complicato. L'impegno del paziente sarà compensato dalle numerose altre settimane libere con una sola dialisi settimanale. E' disponibile un foglio Excel per i calcoli di cui sopra.

\section{Discussione}

La generazione di urea pubblicata da Maroni e Mitch oltre 30 anni fa rimane ancora il mezzo più valido per ricavare indirettamente dalla PCR l'assunzione proteico del paziente con CKD5 e CK5D quando il VFG (calcolato con la media della clearance dell'urea e della creatinina) si aggira su valori molto bassi tenendo conto della superficie corporea. Tale elemento di normalizzazione si è dimostrato molto efficace in popolazioni italiane (Sardegna) ed estere (Cina) ove il peso forma medio di un paziente in emodialisi varia tra i $55-60 \mathrm{~kg}$, specie nel sesso femminile. Tra gli aspetti innovativi che possono essere introdotti nell'applicare tale metodologia si ricordano: la possibilità di portare a bilancio neutro l'input/output dei fosfati che può ripercuotersi positivamente sugli eventi cardio-vascolari anche letali ${ }^{19,20}$; la sempre crescente importanza di eliminare con la FRR le Protein Bound Uremic Toxins (PBUT) che legate all'albumina possono essere eliminate solo da una FRR anche minima. ${ }^{21,23}$ 
Sono sempre più presenti in letteratura evidenze di quanto possa essere importante riequilibrare la disbiosi del microbiota nell'intestino dell'uremico con CKD avanzata, che può condurre ad una regolarizzazione della permeabilità della parete intestinale. Infatti nell'uremia l'alterazione di tale permeabilità può favorire un eccessivo assorbimento ematico di citochine, lipopolisaccaridi e PBUT. A questo riguardo si segnala fortemente di evitare la somministrazione di prodotti a base di L-carnitina. ${ }^{24}$

Per concludere occorre accennare a quel percorso che può essere definito dialisi decrementale. Infatti i nefrologi che focalizzano la loro attenzione sulla diuresi residua incapperanno in pazienti in emodialisi trisettimanale che continuano a conservare una diuresi $>1000 \mathrm{~mL} / \mathrm{die}$. In questo caso sarebbe opportuno decrementare prudentemente la dose depurativa diminuendo così la eccessiva "dose" dialitica e le numerose patologie ad essa correlate. Nella nostra esperienza quasi ventennale non ci siamo mai trovati di fronte a sorprese cliniche spiacevoli perché con una buona dose dialitica ed una buona collaborazione tra tutto il personale sanitario ed il paziente il programma CDDP diventa facilmente gestibile nella routine di tutti i giorni.

\section{Conclusioni}

Si presuppone che in qualsiasi centro esistano pazienti con una diuresi 'interessante'. Pertanto si ritiene che un maggior impegno clinico possa evitare una dose di dialisi eccessiva poiché la FRR e la diuresi residua hanno notevoli potenzialità depurative. Sappiamo inoltre che la prescrizione dietetica ipoproteica, oltre che rallentare di per se stessa il decadimento della funzione renale residua, evita incongrue generazioni di urea e di metaboliti di maggiori dimensioni ben più tossici sull'asse cuore-rene. Riteniamo quindi che questo programma possa essere applicabile in tutti i centri e possa essere molto utile per riavvicinare i vecchi e giovani nefrologi alla biofisica e biochimica dell'uremia e della dialisi offrendo ai pazienti una alternativa terapeutica valida.

\section{Dichiarazione di assenza di conflitto di interessi}

Gli Autori dichiarano di non aver alcun conflitto di interesse né aver ricevuto alcun contributo per la preparazione dell'articolo. Non è stata esercitata alcuna influenza intellettuale, commerciale sulla stesura e conclusione del lavoro da parte di Aziende o Enti privati.

\section{Bibliografia}

1. Chandna SM e Farrington K. Residual renal function: considerations on its importance and preservation in dialysis patients. Semin Dial 2004; 17: 196-201.

2. Wang M, Chou J, Chang Y, et al. The role of low protein diet in ameliorating proteinuria and deferring dialysis initiation: what is old and what is new. Panminerva Med 2017; 59: $157-165$.
3. Mathew AT, Fishbane S, Obi Y, et al. Preservation of residual kidney function in hemodialysis patients: reviving an old concept. Kidney Int 2016; 90: 262-71.

4. Locatelli F, Andrulli S, Pontoriero G, Di Filippo S e Bigi MC. Supplemented low-protein and once-weekly hemodialysis. Am J Kidney Dis 1994; 24: 192-204.

5. Morelli E, Baldi R, Barsotti G, et al. Combined therapy for selected chronic uremic patients: infrequent hemodialysis and nutritional management. Nephron 1987; 47: 161-166.

6. Mitch WE e Sapir DG. Evaluation of reduced dialysis frequency using nutritional therapy. Kidney Int 1981; 20: 122-126.

7. Locatelli F, Andrulli S, Pontoriero G, et al. Integrated diet and dialysis programme. Nephrol Dial Transplant 1998; 13 Suppl 6: 132-138.

8. Caria S, Cupisti A, Sau G, et al. The incremental treatment of ESRD: a low-protein diet combined with weekly hemodialysis may be beneficial for selected patients. $B M C$ Nephrol 2014; 15: 172.

9. Bolasco P, Caria S, Egidi MF, et al. Approccio incrementale di inizio dialisi: emodialisi bisettimanale, o monosettimanale associata a dieta ipoproteica-ipofosforica? G Ital Nefrol 2015; 32(6).

10. Knerr K, Füth R, Hemsen P, et al. Chronic inflammation and hemodialysis reduce immune competence of peripheral blood leukocytes in end-stage renal failure patients. Cytokine 2005; 30: 132-138.

11. Macías Núñez JF, Ghais Z, Bustamante J, et al. Evaluation of oxidant-antioxidant balance in patients on maintenance haemodialysis: a comparative study of dialyzers membranes. Nephron Clin Pract 2010; 114: c67-c73.

12. Lysaght MJ, Vonesh EF, Gotch F, et al. The influence of dialysis treatment modality on the decline of remaining renal function. ASAIO trans 1991; 37: 598-604.

13. McKane W, Chandna SM, Tattersall JE, et al. Identical decline of residual renal function in high-flux biocompatible hemodialysis and CAPD. Kidney Int 2002; 61: 256-265.

14. Alipoor E, Hosseinzadeh-Attar MJ, Mahdavi-Mazdeh $\mathrm{M}$, et al. Comparison of malnutrition inflammation score, anthropometry and biochemical parameters in assessing the difference in protein-energy wasting between normal weight and obese patients undergoing haemodialysis. Nutr Diet 2017; 74: 283-290.

15. Kokubo K, Kurihara Y, Kobayashi K, et al. Evaluation of the Biocompatibility of Dialysis Membranes. Blood Purif 2015; 40: 293-297.

16. Bolasco P, Contu A, Meloni P, et al. Microbiological surveillance and state of the art technological strategies for the prevention of dialysis water pollution. Int J Environ Res Public Health 2012; 9: 2758-2571.

17. Maroni BJ, Steinman TI e Mitch WE. A method for estimating nitrogen intake of patients with chronic renal failure. Kidney Int 1985; 27: 58-65.

18. Watson PE, Watson ID e Batt RD. Total body water volumes for adult males and females estimated from simple anthropometric measurements. Am J Clin Nutr 1980; 33: 27-39.

19. Bolasco P, Murtas S, Caria S, et al. The power of phosphaturia in the infrequent hemodialysis. G Ital Nefrol 2017; 34(1). 
20. Bolasco P e Murtas S. Clinical benefits of phosphate control in progression of end stage renal disease. Panminerva Med 2017; 59: 133-138.

21. Deltombe O, Van Biesen W, Glorieux G, et al. Exploring Protein Binding of Uremic Toxins in Patients with Different Stages of Chronic Kidney Disease and during Hemodialysis. Toxins (Basel) 2015; 7: 3933-3946.

22. Wu IW, Hsu KH, Lee CC, et al. P-cresyl sulphate and indoxyl sulphate predict progression of chronic kidney disease. Nephrol Dial Transplant 2011; 26: 938-947.

23. Vanholder R, Schepers E, Pletinck A, et al. The uremic toxicity of indoxyl sulfate and p-cresyl sulfate: a systematic review. J Am Soc Nephrol 2014; 25: 1897-1907.

24. Irie J, Kanno Y, Kikuchi R, et al. L-carnitine improves gastrointestinal disorders and altered the intestinal microbiota in hemodialysis patients. Biosci Microbiota Food Health 2017; 36: 11-16.

\section{Appendice I}

Gli Autori rimangono a disposizione per qualsiasi ulteriore in-formazione o collaborazione all'indirizzo mail pg.bolasco@tin. it.

Il centro di riferimento è la S.C. Territoriale di Nefrologia e Dialisi - ASSL Cagliari.

Via Turati 4/C1

09045 Quartu Sant'Elena

Alla osservazione del Responsabile Stefano Murtas

Questo proposta di protocollo è stata concordemente pubblicata sul primo numero del 2018 del Giornale Italiano di Nefrologia e del Giornale di Tecniche Nefrologiche e Dialitiche. 